\title{
Article
}

\section{Evaluation of convergent series by using finite parts}

\section{Ricardo Estrada}

Department of Mathematics, Louisiana State University, Baton Rouge, LA 70803, U.S.A.; restrada@math.lsu.edu Received: 4 December 2019; Accepted: 21 March 2020; Published: 24 March 2020.

Abstract: We present a method to find the sum of a convergent series based on the computation of Hadamard finite part limits of partial sums. We give several illustrations, the main being the formulas for convergent series of the type $\sum_{n=2}^{\infty} \frac{(-1)^{n} \zeta(n, a) b^{n+k}}{n+k}$, where $\zeta(s, a)$ is Hurwitz zeta function, $|b| \leq|a|, b \neq-a$, and $k \in \mathbb{N}$.

Keywords: Hadamard finite part limits, summation of series, Hurwitz zeta function, digamma function.

MSC: 11M35, 40A25, 41A58.

\section{Introduction}

$\mathbf{T}$ he aim of this article is to present a method for the summation of convergent series by using the finite part of divergent series in the intermediate steps. Using divergent series to find the result of convergent processes is a very old practice as can be seen very clearly by reading chapter 1 of Hardy's book [1] or by consulting classic texts on series such as Bromwich's [2]; a great variety of summability methods have been employed throughout the years [1]. This article differs from older methods, however, in that we employ Hadamard finite part limits in our process. Finite part limits are explained in Sections 3 and 4, and the many examples given should convince the reader that the extraction of the finite part of a series that diverges to infinity is a regularization procedure, not a summability method. Hadamard finite parts of limits and integrals have been employed mainly in the areas of partial differential equations, since they were first defined by Hadamard [3] to find fundamental solutions of hyperbolic partial differential equations, and in the theory of distributions and generalized functions [4,5], where they play a rather important part, mostly because of their relationship with pseudofunctions.

In Section 5, we find the sum of the convergent series

$$
\sum_{n=0}^{\infty}\left\{\ln \left(\frac{n+a+1}{n+a}\right)-\frac{1}{n+a}\right\}
$$

by finding the finite part of the sums of the two divergent series

$$
\sum_{n=0}^{\infty} \ln \left(\frac{n+a+1}{n+a}\right) \quad \text { and } \quad \sum_{n=0}^{\infty} \frac{1}{n+a},
$$

and substracting those results. Next, in Section 6, we consider a more complicated example, namely the convergent series

$$
\sum_{n=0}^{\infty}\left\{(n+a) \ln \left(\frac{n+a+1}{n+a}\right)-1+\frac{1}{2(n+a)}\right\},
$$

where we employ standard ideas such as Abel's summation formula and Stirling's asymptotic approximation to find the finite part of the divergent series encountered.

Sections 7 and 8 contain a more systematic treatment of a class of convergent series that generalize (1) and (3). The main tool of our analysis is the formula

$$
\text { F.p. } \sum_{n=0}^{\infty}(n+a)^{k} \ln (n+a)=-\zeta^{\prime}(-k, a)-H_{k} \zeta(-k, a+1) \text {, }
$$

for the finite part of the divergent series $\sum_{n=0}^{\infty}(n+a)^{k} \ln (n+a)$, where $H_{k}=\sum_{j=1}^{k}(1 / j)$ are the harmonic numbers. 
We are then able to find the sum of certain convergent series involving values of the Hurwitz zeta function, namely, those of the form

$$
v_{k}(a, b)=\sum_{n=2}^{\infty} \frac{(-1)^{n} \zeta(n, a) b^{n+k}}{n+k},
$$

for $|b| \leq|a|, b \neq-a, k \in \mathbb{N}$. Series of this family have received a lot of attention since the times of Euler, who gave the interesting sums

$$
v_{0}(1,1)=\sum_{n=2}^{\infty} \frac{(-1)^{n} \zeta(n)}{n}=\gamma
$$

and

$$
\nu_{0}(2,-1)=\sum_{n=2}^{\infty} \frac{\zeta(n, 2)}{n}=\sum_{n=2}^{\infty} \frac{\zeta(n)-1}{n}=1-\gamma
$$

where $\gamma$ is Euler's constant. Srivastava [6] considers many of these series in an article that contains a very detailed historical account of the contributions of several authors. Recently, Blagouchine [7] and Coppo [8] have given general formulas for the series

$$
v_{k}(1,1)=\sum_{n=2}^{\infty} \frac{(-1)^{n} \zeta(n)}{n+k}
$$

for all $k$, that generalize not only (6) but also the nice Surnayanarayana's formula [9]

$$
\nu_{1}(1,1)=\sum_{n=2}^{\infty} \frac{(-1)^{k} \zeta(n)}{n+1}=\frac{\gamma}{2}+1-\ln \sqrt{2 \pi} .
$$

Interestingly, Surnayanarayana also considers divergent but Cesàro summable sums in [9], as the formula

$$
\sum_{n=2}^{\infty}(-1)^{k} \zeta(n)=1
$$

that can also be found by employing exterior Euler summability [10] and is also ammenable of our analysis. Notice that the series (5) diverges to infinity if $b=-a$. Nevertheless, after reading this article the reader will be able to see that

$$
\text { F.p. } \sum_{n=2}^{\infty} \zeta(n)=-1
$$

\section{Preliminaries}

In this section, we fix the notation employed and recall several useful well known facts; see [11] and [12] for details.

We shall always consider the principal branches of powers and logarithms, $z^{\alpha}$ and $\ln z$, defined for $z \in$ $\mathbb{C} \backslash(-\infty, 0]$. If $a \in \mathbb{C} \backslash(-\infty, 0]$, the Hurwicz zeta function $\zeta(s, a)$ is the analytic continuation to $s \in \mathbb{C} \backslash\{1\}$ of the function

$$
\zeta(s, a)=\sum_{n=0}^{\infty} \frac{1}{(n+a)^{s}}, \quad \text { Re } s>1 .
$$

The zeta function $\zeta(s, a)$ has a simple pole at $s=1$, with residue 1 . If $a=1$ it reduces to the Riemman zeta function, $\zeta(s, 1)=\zeta(s)$. We shall also employ the digamma function $\psi(a)=\Gamma^{\prime}(a) / \Gamma(a)$, the logarithmic derivative of the gamma function, whose Mittag-Leffer expansion is the following

$$
\psi(a)=-\gamma+\sum_{n=0}^{\infty}\left(\frac{1}{n+1}-\frac{1}{n+a}\right), \quad a \neq 0,-1,-2, \ldots,
$$

where $\gamma$ is Euler's constant. The digamma function is actually the finite part - explained in the next section of the negative of the Hurwitz zeta function, $-\zeta(s, a)$, at $s=1$, that is

$$
\psi(a)=-\lim _{s \rightarrow 1}\left(\zeta(s, a)-\frac{1}{s-1}\right)
$$

for $a \in \mathbb{C} \backslash(-\infty, 0]$. Notice also that 


$$
\psi(k)=-\gamma+\sum_{n=1}^{k-1} \frac{1}{n}, \quad k \in\{1,2,3, \ldots\} .
$$

It is easy to see that for $n \geq 1$,

$$
\psi^{(n)}(a)=(-1)^{n+1} n ! \zeta(n+1, a)
$$

and this yields the Taylor expansion

$$
\psi(a+\omega)=\psi(a)+\sum_{n=1}^{\infty}(-1)^{n+1} \zeta(n+1, a) \omega^{n},
$$

which is an absolutely convergent series for $|\omega|<|a|$. The series is divergent for $|\omega|=|a|$ but it is Cesàro summable $^{1}$ in the this circle except for $\omega \neq-a$.

Sometimes, we shall need to use Abel's summation formula [13, Chp. 1],

$$
\sum_{n=1}^{N} a_{n} b_{n}=A_{N} b_{N}-\sum_{n=1}^{N-1} A_{N}\left(b_{n+1}-b_{n}\right)
$$

where $A_{n}=\sum_{j=1}^{n} a_{j}$. We shall also employ Stirling's asymptotic formula [12],

$$
\ln \Gamma(z)=\left(z-\frac{1}{2}\right) \ln z-z+\ln \sqrt{2 \pi}+O\left(\frac{1}{z}\right)
$$

as $z \rightarrow \infty$ in the region $-\pi<\arg z<\pi$.

\section{The finite part of limits}

Let us now recall the notion of the finite part of a limit [13, Section 2.4]. Let $X$ be a topological space, and let $x_{0} \in X$. Suppose $\mathfrak{F}$, the basic functions, is a family of strictly positive functions defined for $x \in V \backslash\left\{x_{0}\right\}$, where $V$ is a neighborhood of $x_{0}$, such that all of them tend to infinity at $x_{0}$ and such that, given two different elements $f_{1}, f_{2} \in \mathfrak{F}$, then $\lim _{x \rightarrow x_{0}} f_{1}(x) / f_{2}(x)$ is either 0 or $\infty$.

Definition 1. Let $G(x)$ be a function defined for $x \in V \backslash\left\{x_{0}\right\}$ with $\lim _{x \rightarrow x_{0}} G(x)=\infty$. The finite part of the limit of $G(x)$ as $x \rightarrow x_{0}$ with respect to $\mathfrak{F}$ exists and equals $A$ if we can write ${ }^{2}(x)=G_{1}(x)+G_{2}(x)$, where $G_{1}$, the infinite part, is a linear combination of the basic functions and where $G_{2}$, the finite part, has the property that the limit $A=\lim _{x \rightarrow x_{0}} G_{2}(x)$ exists. We then employ the notation

$$
\text { F.p. } \lim _{x \rightarrow x_{0}} G(\varepsilon)=A \text {. }
$$

We shall be interested in the Hadamard finite parts at infinity in this article. It is the case when $\mathfrak{F}$ is the family of powers and logarithms.

Definition 2. The Hadamard finite part limit corresponds to the case when $x_{0}=0$ and $\mathfrak{F}$ is the family of functions $x^{-\alpha}|\ln x|^{\beta}$, where $\alpha>0$ and $\beta \geq 0$ or where $\alpha=0$ and $\beta>0$ or when $x_{0}=\infty$ and $\mathfrak{F}$ is the family of functions $x^{\alpha}|\ln x|^{\beta}$, where $\alpha>0$ and $\beta \geq 0$ or where $\alpha=0$ and $\beta>0$. We then use the simpler notations F.p. $\lim _{x \rightarrow 0^{+}} G(x)$ or F.p. $\lim _{x \rightarrow \infty} G(x)$.

Applying the notion of finite part of limits we can define the finite part of integrals at singularities [13, Section 2.4], but in this article, we will be interested in the finite part of series,

$$
\text { F.p. } \sum_{n=n_{0}}^{\infty} a_{n}=\text { F.p. } \lim _{N \rightarrow \infty} \sum_{n=n_{0}}^{N} a_{n} \text {. }
$$

\footnotetext{
Details on summability can be found in $[1,13]$

Such a decomposition, if it exists, is unique since any finite number of elements of $\mathfrak{F}$ has to be linearly independent.
} 
Observe that the finite part of a series gives a finite sum to a series that diverges to infinity; it does not work for oscillatory series. This makes the finite part process very different from summability methods like Abel summability of Cesàro summability. The finite part process is more of a regularization procedure than a summability method [13, Section 2.4].

Needless to say, many of the usual operations that are valid for convergent series may not work for the finite parts. For example, F.p. $\sum_{n=n_{0}+1}^{\infty} a_{n-1}$ does not have to be equal to F.p. $\sum_{n=n_{0}}^{\infty} a_{n}$.

The notion of finite part integrals and its name were introduced by Hadamard [3], who used them in his study of fundamental solutions of partial differential equations. The finite part method has been a very important part of the theory of distributions since its beginnings [5] since it is closely related to the notion of pseudofunction.

\section{Some finite part limits}

In this section, for future reference, we will give the formulas for several useful finite part limits. Our definition says that F.p. $\lim _{N \rightarrow \infty} N^{\beta}=0$ for all $\beta \in \mathbb{R}$. While it is true that

$$
\text { F.p. } \lim _{N \rightarrow \infty}(N+a)^{\beta}=0, \quad \beta \in \mathbb{R} \backslash\{1,2,3, \ldots\} \text {, }
$$

for all $a \in \mathbb{C}$, we have

$$
\text { F.p. } \lim _{N \rightarrow \infty}(N+a)^{k}=a^{k}, \quad k=1,2,3, \ldots
$$

In a similar fashion, while F.p. $\lim _{N \rightarrow \infty} N^{\beta} \ln N=0$ for all $\beta \in \mathbb{R}$, by definition, when we replace $N$ by $N+a$ we have the ensuing formulas.

Lemma 1. If $a \in \mathbb{C}$ and $\beta \in \mathbb{R} \backslash\{1,2,3, \ldots\}$ then

$$
\text { F.p. } \lim _{N \rightarrow \infty}(N+a)^{\beta} \ln (N+a)=0 \text {. }
$$

If $k=1,2,3, \ldots$ then

$$
\text { F.p. } \lim _{N \rightarrow \infty}(N+a)^{k} \ln (N+a)=H_{k} a^{k}
$$

where $H_{k}$ is the harmonic sum

$$
H_{k}=1+\frac{1}{2}+\cdots+\frac{1}{k}
$$

Proof. If $k \in\{1,2,3, \ldots\}$ then

$$
\text { F.p. } \begin{aligned}
\lim _{N \rightarrow \infty}(N+a)^{k} \ln (N+a) & =\text { F.p. } \lim _{N \rightarrow \infty}(N+a)^{k} \ln N+\text { F.p. } \lim _{N \rightarrow \infty}(N+a)^{k} \ln \left(1+\frac{a}{N}\right) \\
& =\text { F.p. } \lim _{N \rightarrow \infty}(N+a)^{k} \ln \left(1+\frac{a}{N}\right),
\end{aligned}
$$

while for $N$ large

$$
(N+a)^{k} \ln \left(1+\frac{a}{N}\right)=\left(\sum_{j=0}^{k}\left(\begin{array}{c}
k \\
j
\end{array}\right) N^{k-j} a^{j}\right) \sum_{n=1}^{\infty}(-1)^{n+1} \frac{a^{n}}{n N^{n}}
$$

so that

$$
\text { F.p. } \lim _{N \rightarrow \infty}(N+a)^{k} \ln \left(1+\frac{a}{N}\right)=\left\{k\left(\begin{array}{l}
k \\
1
\end{array}\right)-\frac{1}{2}\left(\begin{array}{l}
k \\
2
\end{array}\right)+\cdots+\frac{(-1)^{k}}{k}\left(\begin{array}{l}
k \\
k
\end{array}\right)\right\} a^{k} .
$$

Observe now that

$$
k\left(\begin{array}{l}
k \\
1
\end{array}\right)-\frac{1}{2}\left(\begin{array}{l}
k \\
2
\end{array}\right)+\cdots+\frac{(-1)^{k}}{k}\left(\begin{array}{l}
k \\
k
\end{array}\right)=\int_{0}^{1} \frac{1-(1-x)^{k}}{x} \mathrm{~d} x=\int_{0}^{1} \frac{1-t^{k}}{1-t} \mathrm{~d} t=\int_{0}^{1}\left(1+t+\cdots+t^{k-1}\right) \mathrm{d} t=H_{k}
$$

which yields (25).

Next, we would like to consider the finite part limit of several finite sums. We have that $\sum_{n=1}^{\infty} n^{-\beta}=\zeta(\beta)$, a convergent series if $\Re e \beta>1$. We now consider the finite part limit F.p. $\lim _{N \rightarrow \infty} \sum_{n=1}^{N} n^{-\beta}$ for $\Re e \beta \leq 1$; do we obtain $\zeta(\beta)$ ? The answer is yes almost all the time, but not always. 
Lemma 2. If $\Re e \beta \in \mathbb{R} \backslash\{1,0,-1,-3,-5, \ldots\}$ then

$$
\text { F.p. } \lim _{N \rightarrow \infty} \sum_{n=1}^{N} n^{-\beta}=\zeta(\beta) \text {. }
$$

If $\beta=-k=0,1,3,5, \ldots$ then

$$
\text { F.p. } \lim _{N \rightarrow \infty} \sum_{n=1}^{N} n^{-\beta}=\text { F.p. } \lim _{N \rightarrow \infty} \sum_{n=1}^{N} n^{k}=0,
$$

while if $\beta=1$,

$$
\text { F.p. } \lim _{N \rightarrow \infty} \sum_{n=1}^{N} \frac{1}{n}=\gamma \text {. }
$$

The finite part limit F.p. $\lim _{N \rightarrow \infty} \sum_{n=1}^{N} n^{-\beta}$ does not exist if $\Re e \beta \in\{1,0,-1,-3,-5, \ldots\}, \Im m \beta \neq 0$.

Proof. Formula (29) is trivial, while (30) follows from the well known asymptotic relation

$$
\sum_{n=1}^{N} \frac{1}{n}=\ln N+\gamma+O\left(\frac{1}{N}\right)
$$

In the other cases, we just employ the asymptotic expansion

$$
\zeta(s) \sim \sum_{n=1}^{N} \frac{1}{n^{s}}-\frac{N^{1-s}}{1-s}+\zeta(0) N^{-s}-\sum_{q=0}^{\infty} \frac{\zeta(-2 q-1) s(s+1) \cdots(s+2 q)}{(2 q+1) !} N^{-s-2 q-1},
$$

that comes from the Euler-Maclaurin formula.

It is interesting that the formula (28) holds for some negative integers, namely, for $-2,-4,-6, \ldots$ Observe also that a simple modification of the idea of finite part would make (28) hold when $\Re e \beta \in$ $\{1,0,-1,-3,-5, \ldots\}, \Im m \beta \neq 0$, but we shall not need to consider this case in this article.

Similar formulas are obtained if we replace $n$ by $n+a$ in the previous lemma.

Lemma 3. Let $a \in \mathbb{C} \backslash(-\infty, 0]$. If $\Re e \beta \in \mathbb{R} \backslash\{1,0,-1,-3,-5, \ldots\}$ then

$$
\text { F.p. } \lim _{N \rightarrow \infty} \sum_{n=0}^{N}(n+a)^{-\beta}=\zeta(\beta, a) \text {. }
$$

If $\beta=-k=0,1,3,5, \ldots$ then

$$
\text { F.p. } \lim _{N \rightarrow \infty} \sum_{n=0}^{N}(n+a)^{-\beta}=\text { F.p. } \lim _{N \rightarrow \infty} \sum_{n=0}^{N}(n+a)^{k}=a^{k},
$$

while if $\beta=1$,

$$
\text { F.p. } \lim _{N \rightarrow \infty} \sum_{n=0}^{N} \frac{1}{n+a}=-\psi(a) \text {. }
$$
0.

The finite part limit F.p. $\lim _{N \rightarrow \infty} \sum_{n=0}^{N}(n+a)^{-\beta}$ does not exist in case $\Re e \beta \in\{1,0,-1,-3,-5, \ldots\}, \Im m \beta \neq$

Proof. The proof is basically the same as that of the previous lemma if we use the asymptotic expansion (56) instead of (32) and the formula [14]

$$
\sum_{n=0}^{N} \frac{1}{n+a}=\ln (N+a)-\psi(a)+O\left(\frac{1}{N}\right)
$$

that corresponds to (31). 
It is important to point out that while $\zeta(\beta)$ is obtained by putting $a=1$ in $\zeta(\beta, a)$, the formulas in the Lemmas 2 and 3, are not exactly the same: the fact that we obtain $a^{k}$ in (33) and not 0 as in (29) is explained because one sum is from $n=0$ to $N$ while the other from $n=1$ to $N$.

\section{A simple example}

Let us consider the convergent series

$$
\sigma_{0}(a)=\sum_{n=0}^{\infty}\left\{\ln \left(\frac{n+a+1}{n+a}\right)-\frac{1}{n+a}\right\}
$$

where $a$ is a constant, $a \neq 0,-1,-2, \ldots$ We can easily evaluate it by using finite parts,

$$
\sigma_{0}(a)=\text { F.p. } \lim _{N \rightarrow \infty} \sum_{n=0}^{N} \ln \left(\frac{n+a+1}{n+a}\right)-\text { F.p. } \lim _{N \rightarrow \infty} \sum_{n=0}^{N} \frac{1}{n+a},
$$

since we have a telescoping sum,

$$
\sum_{n=0}^{N} \ln \left(\frac{n+a+1}{n+a}\right)=\ln (N+a+1)-\ln a,
$$

which, when we notice that F.p. $\lim _{N \rightarrow \infty} \ln (N+a+1)=0$, gives the finite part limit

$$
\text { F.p. } \lim _{N \rightarrow \infty} \sum_{n=0}^{N} \ln \left(\frac{n+a+1}{n+a}\right)=-\ln a \text { ， }
$$

while from (34) F.p. $\lim _{N \rightarrow \infty} \sum_{n=0}^{N}(n+a)^{-1}=-\psi(a)$. Hence

$$
\sigma_{0}(a)=\psi(a)-\ln a .
$$

There is also a simple alternative evaluation of $\sigma_{0}(a)$, without the use of finite parts, since (13) gives

$$
\begin{aligned}
\int_{0}^{1} \psi(a+\omega) \mathrm{d} \omega & =-\gamma+\sum_{n=0}^{\infty}\left(\frac{1}{n+1}-\int_{0}^{1} \frac{1}{n+a+\omega} \mathrm{d} \omega\right)=-\gamma+\sum_{n=0}^{\infty}\left(\frac{1}{n+1}-\ln \left(\frac{n+a+1}{n+a}\right)\right) \\
& =\psi(a)+\sum_{n=0}^{\infty}\left(\frac{1}{n+a}-\ln \left(\frac{n+a+1}{n+a}\right)\right),
\end{aligned}
$$

or

$$
\sigma_{0}(a)=\psi(a)-\int_{0}^{1} \psi(a+\omega) \mathrm{d} \omega
$$

and

$$
\int_{0}^{1} \psi(\omega+a) \mathrm{d} \omega=\left.\ln \Gamma(\omega+a)\right|_{0} ^{1}=\ln a .
$$

We also observe that from (17),

$$
v_{0}(a)=\sum_{n=2}^{\infty} \frac{(-1)^{n} \zeta(n, a)}{n}=\int_{0}^{1}(\psi(a+\omega)-\psi(a)) \mathrm{d} \omega=-\sigma_{0}(a) .
$$

If $a=1$, we obtain the well known formula that goes back to Euler,

$$
v_{0}(1)=\sum_{n=2}^{\infty} \frac{(-1)^{n} \zeta(n)}{n}=\gamma .
$$

\section{Another example}

Our next task is to find the sum of the convergent series

$$
\sigma_{1}(a)=\sum_{n=0}^{\infty}\left\{(n+a) \ln \left(\frac{n+a+1}{n+a}\right)-1+\frac{1}{2(n+a)}\right\} .
$$


First, we approximate the sum

$$
S_{N}=\sum_{n=0}^{N} n \ln \left(\frac{n+a+1}{n+a}\right)=\sum_{n=0}^{N} n(\ln (n+a+1)-\ln (n+a))
$$

by employing Abel's summation formula (18),

$$
S_{N}=-\sum_{j=1}^{N} \ln (j+a)+N \ln (N+a+1)=\ln \left(\frac{\Gamma(a+1)}{\Gamma(N+a+1)}\right)+N \ln (N+a+1),
$$

and then Stirling's formula (19), to obtain

$$
S_{N}=\ln \Gamma(a+1)-\left(a+\frac{1}{2}\right) \ln (N+a+1)+N+a+1-\ln \sqrt{2 \pi}+O\left(\frac{1}{N}\right),
$$

and consequently

$$
\text { F.p. } \lim _{N \rightarrow \infty} \sum_{n=0}^{N} n \ln \left(\frac{n+a+1}{n+a}\right)=\ln \Gamma(a+1)+a+1-\ln \sqrt{2 \pi} \text {. }
$$

If we now recall (39), we obtain

$$
\begin{gathered}
\text { F.p. } \sum_{n=0}^{\infty}(n+a) \ln \left(\frac{n+a+1}{n+a}\right)=\ln \Gamma(a+1)+a+1-\ln \sqrt{2 \pi}-a \ln a, \\
\text { and } \sigma_{1}(a)=\ln \Gamma(a+1)+a-\ln \sqrt{2 \pi}-a \ln a-\frac{\psi(a)}{2} .
\end{gathered}
$$

Formulas (49) and (50) allow us to obtain an expression for the sum

$$
v_{1}(a)=\sum_{n=2}^{\infty} \frac{(-1)^{n} \zeta(n, a)}{n+1}
$$

Indeed, (17) yields

$$
v_{1}(a)=\int_{0}^{1} \omega(\psi(a+\omega)-\psi(a)) \mathrm{d} \omega=-\frac{\psi(a)}{2}+\int_{0}^{1} \omega \psi(a+\omega) \mathrm{d} \omega,
$$

and

$$
\begin{aligned}
\int_{0}^{1} \omega \psi(a+\omega) \mathrm{d} \omega & =\int_{0}^{1} \omega\left\{-\gamma+\sum_{n=0}^{\infty}\left(\frac{1}{n+1}-\frac{1}{n+a+\omega}\right)\right\} \mathrm{d} \omega \\
& =- \text { F.p. } \lim _{N \rightarrow \infty} \int_{0}^{1} \sum_{n=0}^{N} \frac{\omega}{n+a+\omega} \mathrm{d} \omega \\
& =\text { F.p. } \lim _{N \rightarrow \infty} \int_{0}^{1} \sum_{n=0}^{N}\left\{\frac{n+a}{n+a+\omega}-1\right\} \mathrm{d} \omega \\
& =-1+\text { F.p. } \lim _{N \rightarrow \infty} \sum_{n=0}^{N}(n+a) \ln \left(\frac{n+a+1}{n+a}\right) \\
& =\ln \Gamma(a+1)+a-\ln \sqrt{2 \pi}-a \ln a
\end{aligned}
$$

so that $v_{1}(a)=\sigma_{1}(a)$, that is,

$$
v_{1}(a)=-\frac{\psi(a)}{2}+\ln \Gamma(a+1)+a-\ln \sqrt{2 \pi}-a \ln a .
$$

Notice that if $a=1$ we obtain Surnayanarana's formula [9]

$$
\sum_{n=2}^{\infty} \frac{(-1)^{n} \zeta(n)}{n+1}=\frac{\gamma}{2}+1-\ln \sqrt{2 \pi}
$$


while $a=2$ yields the result [6]

$$
\sum_{n=2}^{\infty} \frac{(-1)^{n}(\zeta(n)-1)}{n+1}=\frac{\gamma}{2}+\frac{3}{2}-\ln \sqrt{8 \pi}
$$

\section{The functions $\varphi_{k}(a)$}

The Euler-Maclaurin formula yields the asymptotic development

$$
\begin{aligned}
\zeta(s, a) \sim & \sum_{n=0}^{N} \frac{1}{(n+a)^{s}}-\frac{(N+a)^{1-s}}{1-s}+\zeta(0)(N+a)^{-s} \\
& -\sum_{q=0}^{\infty} \frac{\zeta(-2 q-1) s(s+1) \cdots(s+2 q)}{(2 q+1) !}(N+a)^{-s-2 q-1},
\end{aligned}
$$

as $N \rightarrow \infty$. Differentiation of (56) gives the expansion

$$
\begin{aligned}
& \sum_{n=0}^{N} \frac{\ln (n+a)}{(n+a)^{s}} \sim-\zeta^{\prime}(s, a)-\frac{(N+a)^{1-s}}{(1-s)^{2}}+\frac{(N+a)^{1-s} \ln (N+a)}{1-s}-\zeta(0)(N+a)^{-s} \ln (N+a) \\
& -\sum_{q=0}^{\infty} \frac{\zeta(-2 q-1) s(s+1) \cdots(s+2 q)}{(2 q+1) !}(N+a)^{-s-2 q-1}\left(\frac{1}{s}+\cdots+\frac{1}{s+2 q}-\ln (N+a)\right) .
\end{aligned}
$$

Let us define

$$
\varphi_{k}(a)=\text { F.p. } \lim _{N \rightarrow \infty} \sum_{n=0}^{N}(n+a)^{k} \ln (n+a), \quad k \in \mathbb{N} .
$$

We may evaluate $\varphi_{0}(a)$ in two ways, one using Stirlings formula (19),

$$
\begin{aligned}
\varphi_{0}(a) & =\text { F.p. } \lim _{N \rightarrow \infty}[\ln \Gamma(N+a+1)-\ln \Gamma(a)] \\
& =\text { F.p. } \lim _{N \rightarrow \infty}\left[\left(N+a+\frac{1}{2}\right) \ln (N+a+1)-(N+a+1)+\ln \sqrt{2 \pi}-\ln \Gamma(a)\right] \\
& =\ln \sqrt{2 \pi}-\ln \Gamma(a),
\end{aligned}
$$

the other using (57) that gives $\varphi_{0}(a)=-\zeta^{\prime}(0, a)$, so that

$$
\varphi_{0}(a)=-\zeta^{\prime}(0, a)=\ln \left(\frac{\sqrt{2 \pi}}{\Gamma(a)}\right) .
$$

Next we consider $\varphi_{1}(a)$. We have from (57) that $\varphi_{1}(a)$ is given as the finite part of the limit of

$$
-\zeta^{\prime}(-1, a)+\frac{(N+a)^{2}}{4}-\frac{(N+a)^{2} \ln (N+a)}{2}+\frac{(N+a) \ln (N+a)}{2}+\frac{1}{12}-\frac{\ln (N+a)}{12},
$$

as $N \rightarrow \infty$. Hence using the Lemma 1 we obtain

$$
\varphi_{1}(a)=-\zeta^{\prime}(-1, a)+\frac{a^{2}}{2}+\frac{a}{2}+\frac{1}{12} .
$$

More generally, we have the following formula.

Proposition 1. If $k \geq 1$ then

$$
\varphi_{k}(a)=-\zeta^{\prime}(-k, a)-H_{k} \zeta(-k, a+1),
$$

where $H_{k}$ are the harmonic numbers (26).

Proof. It is clear from (57) that

$$
\varphi_{k}(a)=-\zeta^{\prime}(-k, a)+P_{k+1}(a)
$$


where $P_{k+1}$ is a certain polynomial of degree $k+1$. Let us now consider the difference

$$
\begin{aligned}
\varphi_{k}(a+1)-\varphi_{k}(a) & =\left(\zeta^{\prime}(-k, a)-\zeta^{\prime}(-k, a+1)\right)+\left(P_{k+1}(a+1)-P_{k+1}(a)\right) \\
& =-a^{k} \ln a+\left(P_{k+1}(a+1)-P_{k+1}(a)\right) .
\end{aligned}
$$

We also have

$$
\begin{aligned}
\varphi_{k}(a+1)-\varphi_{k}(a) & =\text { F.p. } \lim _{N \rightarrow \infty}\left(\sum_{n=0}^{N}(n+a+1)^{k} \ln (n+a+1)-\sum_{n=0}^{N}(n+a)^{k} \ln (n+a)\right) \\
& =\text { F.p. } \lim _{N \rightarrow \infty}(N+a+1)^{k} \ln (N+a+1)-a^{k} \ln a \\
& =H_{k}(a+1)^{k}-a^{k} \ln a,
\end{aligned}
$$

because of (25). Consequently, $P_{k+1}$ is the a polynomial solution of the equation

$$
P_{k+1}(a+1)-P_{k+1}(a)=H_{k}(a+1)^{k} .
$$

Actually it is the only polynomial solution that satisfies the initial condition

$$
P_{k+1}(0)=-H_{k} \zeta(-k),
$$

since $P_{k+1}(0)$ is the constant term in the polynomial $P_{k+1}(a)$, and (57) tell us that that constant term is precisely $-H_{k} \zeta(-k)$ (that vanishes if $k$ is even).

Furthermore, the Bernoulli polynomials $B_{k}(a)$ satisfy $B_{k}(x+1)-B_{k}(x)=k x^{k-1}, B_{k}(0)=B_{k}(1)=$ $-k \zeta(1-k)$ [11]. Hence $H_{k} B_{k+1}(a+1) /(k+1)$ is another polynomial solution of the initial value problem for the difference equation. Therefore

$$
P_{k+1}(a)=\frac{H_{k}}{k+1} B_{k+1}(a+1) .
$$

Formula (62) is obtained since

$$
\zeta(-k, a)=-\frac{B_{k+1}(-k, a)}{k+1}
$$

as is well known [11].

When $a=1$ it is convenient to consider a variation of the function $\varphi_{k}(1)$.

Proposition 2. If $k \in \mathbb{N}$ then

$$
\text { F.p. } \lim _{N \rightarrow \infty} \sum_{n=1}^{N} n^{k} \ln n=-\zeta^{\prime}(-k)-H_{k} \zeta(-k) \text {. }
$$

Proof. Indeed, using the Lemma 1, we obtain

$$
\text { F.p. } \begin{aligned}
\lim _{N \rightarrow \infty} \sum_{n=1}^{N} n^{k} \ln n & =\varphi_{k}(1)-\text { F.p. } \lim _{N \rightarrow \infty}(N+1)^{k} \ln (N+1) \\
& =-\zeta^{\prime}(-k)-H_{k} \zeta(-k, 2)-H_{k}=-\zeta^{\prime}(-k)-H_{k} \zeta(-k) \text { as required. }
\end{aligned}
$$

8. The series $\sum_{n=2}^{\infty}(-1)^{n} \zeta(n, a) b^{n+k} /(n+k)$

We now employ finite part limits to find a formula for the sum of some general convergent series.

Proposition 3. Let $a \in \mathbb{C} \backslash(-\infty, 0]$ and let $|b| \leq|a|, b \neq-a$. Then for $k \in \mathbb{N}$

$$
\begin{aligned}
& \sum_{n=2}^{\infty} \frac{(-1)^{n} \zeta(n, a) b^{n+k}}{n+k}=\frac{-\psi(a) b^{k+1}}{k+1}-\sum_{j=0}^{k-1} \frac{(-1)^{j} a^{j} b^{k-j}}{k-j}+\sum_{j=0}^{k-1}\left(\begin{array}{c}
k \\
j
\end{array}\right)(-1)^{j} b^{k-j}\left(\zeta^{\prime}(-j, a+b)\right. \\
& \left.+H_{j} \zeta(-j, a+b+1)\right)+(-1)^{k}\left(\zeta^{\prime}(-k, a+b)+H_{k} \zeta(-k, a+b+1)-\zeta^{\prime}(-k, a)-H_{k} \zeta(-k, a+1)\right) .
\end{aligned}
$$


Proof. Indeed, from (17), we obtain

$$
\begin{aligned}
\sum_{n=2}^{\infty} \frac{(-1)^{n} \zeta(n, a) b^{n+k}}{n+k} & =\int_{0}^{b}(-\psi(a)+\psi(a+\omega)) \omega^{k} \mathrm{~d} \omega \\
& =\frac{-\psi(a) b^{k+1}}{k+1}+\int_{0}^{b}\left(-\gamma \omega^{k}+\sum_{n=0}^{\infty}\left(\frac{\omega^{k}}{n+1}-\frac{\omega^{k}}{n+a+\omega}\right)\right) \mathrm{d} \omega \\
& =\frac{-\psi(a) b^{k+1}}{k+1}-\text { F.p. } \lim _{N \rightarrow \infty} \sum_{n=0}^{N} \int_{0}^{b} \frac{\omega^{k}}{n+a+\omega} \mathrm{d} \omega .
\end{aligned}
$$

Observe now that

$$
\frac{\omega^{k}}{n+a+\omega}=\omega^{k-1}-(n+a) \omega^{k-2}+\cdots+(-1)^{k-1}(n+a)^{k-1}+(-1)^{k} \frac{(n+a)^{k}}{n+a+\omega},
$$

which yields

$$
\begin{aligned}
\int_{0}^{b} \frac{\omega^{k}}{n+a+\omega} \mathrm{d} \omega= & \frac{b^{k}}{k}-\frac{(n+a) b^{k-1}}{k-1}+\cdots+(-1)^{k-1}(n+a)^{k-1} b \\
& +(-1)^{k}(n+a)^{k}(\ln (n+a+b)-\ln (n+a)) .
\end{aligned}
$$

Therefore

$$
\begin{aligned}
& \text { F.p. } \lim _{N \rightarrow \infty} \sum_{n=0}^{N} \int_{0}^{b} \frac{\omega^{k}}{n+a+\omega} \mathrm{d} \omega=\sum_{j=0}^{k-1} \frac{(-1)^{j} a^{j} b^{k-j}}{k-j}+(-1)^{k} \\
& \text { F.p. } \lim _{N \rightarrow \infty} \sum_{n=0}^{N}(n+a)^{k}(\ln (n+a+b)-\ln (n+a)) .
\end{aligned}
$$

If we notice now that

$$
\begin{gathered}
\text { F.p. } \lim _{N \rightarrow \infty} \sum_{n=0}^{N}(n+a)^{k} \ln (n+a+b)=\sum_{j=0}^{k}\left(\begin{array}{l}
k \\
j
\end{array}\right)(-b)^{k-j} \varphi_{j}(a+b) \\
=\sum_{j=0}^{k}\left(\begin{array}{l}
k \\
j
\end{array}\right)(-b)^{k-j}\left(-\zeta^{\prime}(-j, a+b)-H_{j} \zeta(-j, a+b+1)\right)
\end{gathered}
$$

and substitute, we obtain (69).

The cases, when $b= \pm 1$ are worth recording. Let us observe that

$\zeta^{\prime}(-k, a+1)+H_{k} \zeta(-k, a+2)-\zeta^{\prime}(-k, a)-H_{k} \zeta(-k, a+1)=\varphi_{k}(a+1)-\varphi_{k}(a)=H_{k}(a+1)^{k}-a^{k} \ln a$

to obtain

$$
\begin{aligned}
\sum_{n=2}^{\infty} \frac{(-1)^{n} \zeta(n, a)}{n+k} & =\frac{-\psi(a)}{k+1}-\sum_{j=0}^{k-1} \frac{(-1)^{j} a^{j}}{k-j}+\sum_{j=0}^{k-1}\left(\begin{array}{c}
k \\
j
\end{array}\right)(-1)^{j}\left(\zeta^{\prime}(-j, a+1)+H_{j} \zeta(-j, a+2)\right) \\
& +(-1)^{k}\left(a^{k} \ln a-H_{k}(a+1)^{k}\right)
\end{aligned}
$$

and

$$
\begin{aligned}
\sum_{n=2}^{\infty} \frac{\zeta(n, a)}{n+k} & =\frac{(-1)^{k} \psi(a)}{k+1}-\sum_{j=0}^{k-1} \frac{a^{j}}{k-j}+\sum_{j=0}^{k-1}\left(\begin{array}{l}
k \\
j
\end{array}\right)\left(\zeta^{\prime}(-j, a-1)+H_{j} \zeta(-j, a)\right) \\
& +H_{k} a^{k}-(a-1)^{k} \ln (a-1) .
\end{aligned}
$$

If we put $a=1$ in (75), we obtain

$$
v_{k}=\sum_{n=2}^{\infty} \frac{(-1)^{n} \zeta(n)}{n+k}=\frac{\gamma}{k+1}-\sum_{j=0}^{k-1} \frac{(-1)^{j}}{k-j}+\sum_{j=0}^{k-1}\left(\begin{array}{l}
k \\
j
\end{array}\right)(-1)^{j}\left(\zeta^{\prime}(-j)+H_{j} \zeta(-j, 3)\right)+(-1)^{k+1} H_{k} 2^{k}
$$


from where one may recover the formula for $v_{k}$ given by Coppo [8], who rewrote the formula given earlier by Blagouchine [7]. On the other hand, setting $a=2$ in (76) gives

$$
\sum_{n=2}^{\infty} \frac{\zeta(n)-1}{n+k}=\frac{-\gamma+1}{k+1}-\sum_{j=0}^{k-1} \frac{(-1)^{j} 2^{j}}{k-j}+\sum_{j=0}^{k-1}\left(\begin{array}{l}
k \\
j
\end{array}\right)(-1)^{j}\left(\zeta^{\prime}(-j)+H_{j}(\zeta(-j)-1)\right)+H_{k} 2^{k}
$$

The case $k=0$,

$$
\sum_{n=2}^{\infty} \frac{\zeta(n)-1}{n}=-\gamma+1
$$

goes back to Euler, while the case $k=1$ can be found in Srivastava [6]

$$
\sum_{n=2}^{\infty} \frac{\zeta(n)-1}{n+1}=-\frac{\gamma}{2}-\ln \sqrt{2 \pi}+\frac{3}{2}
$$

We would also like to point out that by adding and substracting (75) and (76), we can derive formulas for series involving only even or only odd arguments of the Hurwitz zeta function. For example

$$
\begin{gathered}
\sum_{q=2}^{\infty} \frac{\zeta(2 q, a)}{q}=\ln a-\ln (a-1), \\
\sum_{q=2}^{\infty} \frac{\zeta(2 q+1, a)}{2 q+1}=\psi(a)-\ln a-\ln (a-1), \\
\sum_{q=2}^{\infty} \frac{\zeta(2 q, a)}{2 q+1}=\ln \Gamma(a+1)-\ln \sqrt{2 \pi}+a-\frac{1}{2}+\frac{a}{2} \ln a-\frac{(a-1)}{2} \ln (a-1),
\end{gathered}
$$

and

$$
\sum_{q=2}^{\infty} \frac{\zeta(2 q+1, a)}{q+1}=\psi(a)-1-a \ln a+(a-1) \ln (a-1) .
$$

Acknowledgments: The author would like to express their thanks to the referee for his useful remarks.

Conflicts of Interest: "The author declares no conflict of interest."

\section{References}

[1] Hardy, G.H. (1949). Divergent Series. Clarendon Press.

[2] Bromwich, T. J. I'a. (1991). An Introduction to the Theory of Infinite Series. Third edition. Chelsea.

[3] Hadamard, J. (1952). Lectures on Cauchy's Problem in Linear Differential Equations. Dover.

[4] Kanwal, R.P. (2004). Generalized Functions: Theory and Technique. Third Edition. Birkhäuser.

[5] Schwartz, L. (1966). Thèorie des Distributions. Second edition. Hermann.

[6] Srivastava, H. M. (1988). Sums of certain series of the Riemann zeta function, Journal of Mathematical Analysis and Applications, 134, 129-140.

[7] Blagouchine, I.V. (2016). Two series expansions for the logarithm of the gamma function involving Stirling numbers and containing only rational coefficients for certain arguments related to $\pi^{-1}$. Journal of Mathematical Analysis and Applications, 442, 404-434.

[8] Coppo, M.-A. (2019). A note on some alternating series involving zeta and multiple zeta values. Journal of Mathematical Analysis and Applications, 475, 1831-1841.

[9] Suryanarayana, D. (1974). Sums of the Riemann zeta function, Mathematics Student 42, 141-143.

[10] Estrada, R. \& Vindas, J. (2012). Exterior Euler summability, Journal of Mathematical Analysis and Applications, 388, 48-60.

[11] Apostol. P. (1976). Introduction to Analytic Number Theory. Springer Verlag.

[12] Lebedev, N. N. (1972). Special Functions and their Applications. Dover.

[13] Estrada, R. \& Kanwal, R.P. (2002). A Distributional Approach to Asymptotics. Theory and Applications. Second edition. Birkhäuser.

[14] Bracken, P. (2019). On a class of parameter dependent series generalizing Euler's constant. European. Journal of Pure and Applied Mathematics, 12, 1-13. 
(C) 2020 by the authors; licensee PSRP, Lahore, Pakistan. This article is an open access article distributed under the terms and conditions of the Creative Commons Attribution (CC-BY) license (http://creativecommons.org/licenses/by/4.0/). 